\title{
Rainbow path and color degree in edge colored graphs
}

\author{
Anita Das \\ S. V. Subrahmanya \\ P. Suresh \\ E-Comm Research Lab \\ Education \& Research, Infosys Limited \\ Bangalore, India \\ (anita_das01, subrahmanyasv, suresh_p01)@infosys.com
}

Submitted: Oct 1, 2013; Accepted: Feb 12, 2014; Published: Feb 28, 2014

Mathematics Subject Classifications: 05C15, 05C38

\begin{abstract}
Let $G$ be an edge colored graph. A rainbow path in $G$ is a path in which all the edges are colored with distinct colors. Let $d^{c}(v)$ be the color degree of a vertex $v$ in $G$, i.e. the number of distinct colors present on the edges incident on the vertex $v$. Let $t$ be the maximum length of a rainbow path in $G$. Chen and Li (2005) showed that if $d^{c} \geqslant k(k \geqslant 8)$, for every vertex $v$ of $G$, then $t \geqslant\left\lceil\frac{3 k}{5}\right\rceil+1$. Unfortunately, the proof by Chen and Li is very long and comes to about 23 pages in the journal version. Chen and Li states in their paper that it was conjectured by Akira Saito, that $t \geqslant\left\lceil\frac{2 k}{3}\right\rceil$. They also state in their paper that they believe $t \geqslant k-c$ for some constant $c$.

In this note, we give a short proof to show that $t \geqslant\left\lceil\frac{3 k}{5}\right\rceil$, using an entirely different method. Our proof is only about 2 pages long. The draw-back is that our bound is less by 1 , than the bound given by Chen and Li. We hope that the new approach adopted in this paper would eventually lead to the settlement of the conjectures by Saito and/or Chen and Li.
\end{abstract}

Keywords: edge colored graphs; rainbow path; color degree.

\section{Introduction}

Given a graph $G=(V, E)$, a map $c: E \rightarrow N(N$ is the set of non-negative integers $)$ is called an edge coloring of $G$. A graph $\mathrm{G}$ with such a coloring $c$ is called an edge colored graph. We denote the color of an edge $e \in E(G)$ by color $(e)$. For a vertex $v$ of $G$, the color neighborhood $C N(v)$ of $v$ is defined as the set $\{\operatorname{color}(e) \mid e$ is incident on $v\}$ and the color degree of $v$, denoted by $d^{c}(v)$ is defined to be $d^{c}(v)=|C N(v)|$. 
A path in an edge colored graph with no two edges sharing the same color is called a rainbow path. Similarly, a cycle in an edge colored graph is called a rainbow cycle if no two edges of the cycle share the same color. A survey on rainbow paths, cycles and other rainbow sub-graphs can be found in [5]. Several theorems and conjectures on rainbow cycles can be found in a paper by Akbari, Etesami, Mahini and Mahmoody in [1].

Let $t$ denote the length of the maximum length rainbow path in $G$. In [2], Chen and Li studied the maximum length rainbow path problem in edge-colored graphs and proved that if $G$ is an edge colored graph with $d^{c}(v) \geqslant k(k \geqslant 8)$, for every vertex $v$ of $G$, then $G$ has a rainbow path of length at least $\left\lceil\frac{3 k}{5}\right\rceil+1$. Chen and Li state in their paper that it was conjectured by Akira Saito, that $t \geqslant\left\lceil\frac{2 k}{3}\right\rceil$. They also state in their paper that they believe $t \geqslant k-c$ for some constant $c$, after showing an example where the rainbow path cannot be more than $k-1$.

In this note, we give a short proof to show that $t \geqslant\left\lceil\frac{3 k}{5}\right\rceil$, using an entirely different method. Our proof is only about 2 pages long. The draw-back is that our bound is less by 1 , than the bound given by Chen and Li. We hope that the new approach adopted in this paper would eventually lead to the settlement of the conjectures by Saito and/or Chen and Li.

\subsection{Preliminaries}

All graphs considered in this paper are finite, simple and undirected. A graph is a tuple $(V, E)$, where $V$ is a finite set of vertices and $E$ is the set of edges. For a graph $G$, we use $V(G)$ and $E(G)$ to denote its vertex set and edge set, respectively. The neighborhood $N(v)$ of a vertex $v$ is the set of vertices adjacent to $v$ but not including $v$. The degree of a vertex $v$ is $d_{v}=|N(v)|$. A path is a non-empty graph $P=(V, E)$ of the form $V=\left\{p_{1}, p_{2}, \ldots, p_{k}\right\}$ and $E=\left\{\left(p_{1}, p_{2}\right),\left(p_{2}, p_{3}\right), \ldots,\left(p_{k-1}, p_{k}\right)\right\}$, which we usually denote by the sequence $\left\{p_{1}, p_{2}, \ldots, p_{k}\right\}$. The length of a path is its number of edges. If $P=$ $\left\{p_{1}, p_{2}, \ldots, p_{k}\right\}$ is a path, then the graph $C$ with $V(C)=V(P)$ and $E(C)=P \cup\left\{\left(p_{k}, p_{1}\right)\right\}$ is a cycle, and $|E(C)|$ is the length of $C$. We represent this cycle by the cyclic sequence of its vertices, for example $C=\left\{p_{1}, p_{2}, \ldots, p_{k}, p_{1}\right\}$.

\section{Proof of the main results}

Let $G$ be an edge colored graph with $d^{c}(v) \geqslant k$, for every vertex $v$ of $G$ and $t$ be the maximum length rainbow path in $G$. Let $C$ denote the set of colors used in the edge coloring of $G$. The following lemma ensures a rainbow path of length $\left\lceil\frac{k+1}{2}\right\rceil$ starting from any vertex in an edge colored graph.

Lemma 2.1. Let $G$ be an edge colored graph and $d^{c}(v) \geqslant k$, for every $v \in V(G)$. Then, given any vertex $x$ in $G$ there exists a rainbow path of length at least $\left\lceil\frac{k+1}{2}\right\rceil$ starting from $x$.

Proof. Let $P$ be a rainbow path in $G$ of maximum length, say $t$. Thus $P$ contains $t+1$ vertices, $t$ edges and hence $t$ distinct colors. Let $P=\left\{x=u_{0}, u_{1}, \ldots, u_{t}=y\right\}, U=$ 
$\left\{\operatorname{color}\left(u_{i}, u_{i+1}\right), 0 \leqslant i \leqslant t-1\right)$ and $U^{c}=C \backslash U$. Select a subset $E(x)$ of edges incident on $x$ as follows: Let $\left(x, u_{1}\right) \in E(x)$. Now, select $k-1$ more edges incident on $x$ and add to $E(x)$ so that all the $k$ edges in $E(x)$ have different colors. Clearly it is possible to do this, since $d^{c}(x) \geqslant k$.

Let $N_{P}(x)=\left\{u_{i}: 1 \leqslant i \leqslant t\right.$ and $\left.\left(x, u_{i}\right) \in E(x)\right\}, N_{P^{c}}(x)=\{a \in V(G) \backslash V(P)$ : $(x, a) \in E(x)\}$.

Claim. If $z \in N_{P^{c}}(x)$, then $\operatorname{color}(x, z) \in U-\left\{\operatorname{color}\left(x, u_{1}\right)\right\}$.

Suppose that $\operatorname{color}(x, z) \in U^{c}$. As $z \notin V(P), P^{\prime}=\left\{z, x=u_{0}, u_{1}, \ldots, u_{t}=y\right\}$ is a path. Moreover, $P^{\prime}$ is a rainbow path as $\operatorname{color}(x, z) \in U^{c}$. Now $P^{\prime}$ is a rainbow path in $G$ of length $t+1$. This is a contradiction to the fact that $t$ is the length of the maximum length rainbow path in $G$. Also it is obvious that $\operatorname{color}(x, z) \neq \operatorname{color}\left(x, u_{1}\right)$. Hence the claim is true.

From the above claim we infer that $N_{P^{C}}(x)|\leqslant| U \mid-1=t-1$. So, $\left|N_{P}(x)\right| \geqslant k-(t-1)$. But, number of vertices in $P$ excluding $x$ is $t$. So, $k-(t-1) \leqslant t$. Hence, $t \geqslant \frac{k+1}{2}$. Since $t$ is an integer, we have $t \geqslant\left\lceil\frac{k+1}{2}\right\rceil$.

The following lemma ensures that if the maximum length of a rainbow path is small enough, then we can convert the maximum rainbow path into a rainbow cycle by some simple modifications.

Lemma 2.2. Let $G$ be an edge colored graph and $d^{c}(v) \geqslant k$, for every $v \in V(G)$. Let $t$ be the length of the maximum length rainbow path in $G$. If $t<\left\lceil\frac{3}{5} k\right\rceil$, then $G$ contains a rainbow cycle of length $(t+1)$.

Proof. Assume for contradiction that there is no rainbow cycle of length $t+1$ in $G$. Let $P=\left\{u_{0}(=x), u_{1}, u_{2}, \ldots, u_{t}(=y)\right\}$ be a rainbow path of length $t$ in $G$. Let $U=$ $\left\{\operatorname{color}\left(u_{i}, u_{i+1}\right), 0 \leqslant i \leqslant t-1\right\}$ and $U^{c}=C \backslash U$, where $C$ is the set of colors used to color the edges of $G$. Clearly $|U|=t$. Let $T_{x}=\left\{u_{i}: 0 \leqslant i \leqslant t,\left(x, u_{i}\right) \in E(G)\right.$ and $\left.\operatorname{color}\left(x, u_{i}\right) \in U^{c}\right\}$ and let $T_{y}=\left\{u_{i}: 0 \leqslant i \leqslant t,\left(y, u_{i}\right) \in E(G)\right.$ and color $\left.\left(y, u_{i}\right) \in U^{c}\right\}$.

First note that, $\left|\left\{(x, z) \in E(G): \operatorname{color}(x, z) \in U^{c}\right\}\right| \geqslant k-t$. Moreover, if $(x, z) \in E(G)$ with $\operatorname{color}(x, z) \in U^{c}$, then $z \in V(P)$, i.e., $z=u_{i}$ for some $1 \leqslant i \leqslant t$, since otherwise we would have a rainbow path of length $t+1$ in $G$. It follows that $\left|T_{x}\right| \geqslant k-t$. By a similar argument, we get $\left|T_{y}\right| \geqslant k-t$. Note that $u_{0}, u_{1} \notin T_{x}$ since $u_{0}=x$ and $\operatorname{color}\left(x, u_{1}\right) \in U$. Also, $u_{t} \notin T_{x}$, since if $\left(x, u_{t}\right)$ is an edge and is colored using a color from $U^{c}$, then we already have a $t+1$ length rainbow cycle, contrary to the assumption. So, we can write $T_{x}=\left\{u_{i}: 2 \leqslant i \leqslant t-1\right.$ and $\left.\operatorname{color}\left(x, u_{i}\right) \in U^{c}\right\}$. By similar reasoning, we can write, $T_{y}=\left\{u_{i}: 1 \leqslant i \leqslant t-2\right.$ and $\left.\operatorname{color}\left(y, u_{i}\right) \in U^{c}\right\}$. Define $M_{x}=\left\{u_{j}: u_{j+1} \in T_{x}\right\}$.

Observation 1. $\left|M_{x}\right|=\left|T_{x}\right| \geqslant k-t$.

Claim 1. $M_{x} \cap T_{y} \neq \emptyset$.

If possible suppose $M_{x} \cap T_{y}=\emptyset$. Now, $\left|M_{x}\right|+\left|T_{y}\right| \leqslant t-1$, as both $M_{x} \subset V(P)$ and $T_{y} \subset V(P)$ and number of vertices on $P$ excluding $x$ and $y$ is $t-1$. (Note that, $x, y \notin M_{x}$ and $x, y \notin T_{y}$.) As $\left|M_{x}\right| \geqslant k-t$ and $\left|T_{y}\right| \geqslant k-t$ and $M_{x} \cap T_{y}=\emptyset$ by assumption, we have $k-t+k-t \leqslant t-1$. That is, $2 k \leqslant 3 t-1$. So, $t \geqslant \frac{2 k+1}{3}$. This is a contradiction to the fact that $t<\left\lceil\frac{3}{5} k\right\rceil$. Hence Claim 1 is true. 
Claim 2. If $u_{i} \in M_{x} \cap T_{y}$, then color $\left(y, u_{i}\right)=\operatorname{color}\left(x, u_{i+1}\right)$.

Suppose Claim 2 is false. That is, $\exists u_{i} \in M_{x} \cap T_{y}$ such that $\operatorname{color}\left(y, u_{i}\right) \neq \operatorname{color}\left(x, u_{i+1}\right)$. Now consider the cycle: $C L=\left\{x, u_{1}, \ldots, u_{i}, y, u_{t}, u_{t-1}, \ldots, u_{i+1}, x\right\}$. Clearly $C L$ is a rainbow cycle, as color $\left(y, u_{i}\right) \neq \operatorname{color}\left(x, u_{i+1}\right), \operatorname{color}\left(y, u_{i}\right) \in U^{c}$ and $\operatorname{color}\left(x, u_{i+1}\right) \in U^{c}$. Note that the length of $C L$ is $t+1$, as we removed exactly one edge, namely $\left(u_{i}, u_{i+1}\right)$ from $P$ and added two new edges, namely $\left(y, u_{i}\right)$ and $\left(x, u_{i+1}\right)$ to $C L$. So, the length of $C L$ is $t-1+2=t+1$, contradiction to the assumption. Hence, we can infer that if $u_{i} \in M_{x} \cap T_{y}$, then color $\left(y, u_{i}\right)=\operatorname{color}\left(x, u_{i+1}\right)$.

Let $\left.S_{y}=\left\{v \in V(P)-\left(M_{x} \cup\left\{y, u_{t-1}\right\}\right): \operatorname{color}(y, v) \in U\right\}\right\}$.

Observation 2. $\left|M_{x}\right|+\left|T_{y}\right|+\left|S_{y}\right|-\left|M_{x} \cap T_{y}\right| \leqslant t-1$.

Proof: This is because $S_{y}$ is disjoint from $M_{x} \cup T_{y}$ and $S_{y} \cup M_{x} \cup T_{y} \subseteq V(P)-\left\{y, u_{t-1}\right\}$. (Note that $y\left(=u_{t}\right)$ and $u_{t-1}$ do not appear in $M_{x}, T_{y}$ or $S_{y}$.)

We partition the set $M_{x} \cap T_{y}$ as follows. Let $u_{i} \in M_{x} \cap T_{y}$. If $\operatorname{color}\left(u_{i}, u_{i+1}\right)$ appears in one of the edges incident on $y$, then $u_{i} \in A$ otherwise $u_{i} \in B$.

Observation 3. $\left|T_{y}\right| \geqslant k-t+|B|$.

Proof: To see this first note that there are at least $k$ edges of different colors incident on $y$ (as by assumption, color degree of $y$ is at least $k$ )and at most $t-|B|$ of them can get the colors from $U$, since $|B|$ colors in $U$ do not appear on the edges incident on $y$, by the definition of $B$. So, at least $k-t+|B|$ of the edges incident on $y$ have colors from $U^{c}$, and clearly any $w$, such that $(y, w)$ is an edge, colored by a color in $U^{c}$ has to be on $P$, since otherwise we have a longer rainbow path. It follows that $\left|T_{y}\right| \geqslant k-t+|B|$.

Claim 3. If $u_{i} \in A$, then the edge incident on $y$ with color color $\left(u_{i}, u_{i+1}\right)$ has its other end point on the rainbow path $P$. That is, if $w$ is such that $(y, w)$ is an edge and $\operatorname{color}\left(u_{i}, u_{i+1}\right)=\operatorname{color}(y, w)$, then $w \in V(P)$.

Suppose Claim 3 is false. Let $(y, w) \in E(G)$ with $\operatorname{color}(y, w)=\operatorname{color}\left(u_{i}, u_{i+1}\right)$ and $w \notin V(P)$. Now, consider the path: $P^{\prime}=\left\{w, y, u_{t-1}, u_{t-2}, \ldots, u_{i+1}, x\left(=u_{0}\right), u_{1}, \ldots, u_{i}\right\}$. Clearly $P^{\prime}$ is a rainbow path as color $\left(u_{i}, u_{i+1}\right)=\operatorname{color}(y, w)$, the edge $\left(u_{i}, u_{i+1}\right) \notin E\left(P^{\prime}\right)$ and color $\left(u_{i+1}, x\right) \in U^{c}$, since $u_{i} \in M_{x}$. Note that, the length of $P^{\prime}$ is $t+1$. This is a contradiction to the fact that $t$ is the maximum length rainbow path in $G$. Hence Claim 3 is true.

Now, partition $A$ as follows: if $u_{i} \in A$, then by the above claim the edge incident on $y$ with the color $\operatorname{color}\left(u_{i}, u_{i+1}\right)$ has its other end point say $w$, on $P$. If $w \in M_{x}$, then let $u_{i} \in A_{1}$, else $u_{i} \in A_{2}$.

Observation 4. $\left|M_{x} \cap T_{y}\right|=|A|+|B|=\left|A_{1}\right|+\left|A_{2}\right|+|B|$.

Observation 5. $\left|S_{y}\right| \geqslant\left|A_{2}\right|$. To see this, recall that $S_{y}=\left\{v \in V(P)-\left(M_{x} \cup\left\{y, u_{t-1}\right\}\right)\right.$ : $\operatorname{color}(y, v) \in U\}$. By definition of $A_{2}$, for each $u_{i} \in A_{2}$ there exists a unique vertex $w=w\left(u_{i}\right) \in V(P)-M_{x}$ such that $(y, w)$ is an edge and $\operatorname{color}(y, w)=\operatorname{color}\left(u_{i}, u_{i+1}\right) \in U$. Since $u_{i} \in A_{2} \subset M_{x}$, we have $i<t-1$ and thus color $\left(u_{i}, u_{i+1}\right) \neq \operatorname{color}\left(y, u_{t-1}\right)$. Therefore $w\left(u_{i}\right)$ cannot be $y$ or $u_{t-1}$, for any $u_{i} \in A_{2}$. It follows that $\left\{w\left(u_{i}\right): u_{i} \in A_{2}\right\} \subseteq S_{y}$, and therefore we have $\left|S_{y}\right| \geqslant\left|A_{2}\right|$.

Claim 4. $\left|A_{1}\right| \leqslant \frac{\left|M_{x}\right|}{2}$. 
Recall that, for each $u_{i} \in A_{1}$, there is a unique vertex $w=w\left(u_{i}\right)$ such that $(y, w)$ is an edge with $\operatorname{color}\left(u_{i}, u_{i+1}\right)=\operatorname{color}(y, w)$. Moreover, $w \in M_{x}$, by the definition of $A_{1}$ and $A_{1} \cup\left\{w\left(u_{i}\right): u_{i} \in A_{1}\right\} \subseteq M_{x}$. Note that $w\left(u_{i}\right)$ is uniquely defined for $u_{i}$ since it is the end point of the edge incident on $y$ colored with the color of the edge $\left(u_{i}, u_{i+1}\right)$. Moreover, $A_{1} \cap\left\{w\left(u_{i}\right): u_{i} \in A_{1}\right\}=\emptyset$, since $A_{1}$ contains vertices which are end points of edges from $y$, colored by the colors in $U^{c}$ whereas each $w\left(u_{i}\right)$ is the end point of some edge from $y$ which is colored by a color in $U$. It follows that $2\left|A_{1}\right| \leqslant\left|M_{x}\right|$. That is, $\left|A_{1}\right| \leqslant \frac{\left|M_{x}\right|}{2}$, as required.

Now, substituting $k-t+|B|$ for $\left|T_{y}\right|$ (by Observation 3), $\left|A_{2}\right|$ for $\left|S_{y}\right|$ (by Observation 5), and $\left|A_{1}\right|+\left|A_{2}\right|+|B|=\left|M_{x} \cap T_{y}\right|$ (by Observation 4) in the inequality of Observation 2 , and simplifying we get $\left|M_{x}\right|+k-t-\left|A_{1}\right| \leqslant t-1$. Now using $\left|A_{1}\right| \leqslant\left|M_{x}\right| / 2$ (Claim 4) and and simplifying we get $\frac{\left|M_{x}\right|}{2}+k-t \leqslant t-1$. Recall that $\left|M_{x}\right| \geqslant k-t$ (Observation 1 ). Substituting and simplifying we get, $t \geqslant \frac{3 k+2}{5}$. It follows that $t \geqslant\left\lceil\frac{3}{5} k\right\rceil$, contradicting the initial assumption. Hence the Lemma is true.

Theorem 2.3. Let $G$ be an edge colored graph and $d^{c}(v) \geqslant k$, for every $v \in V(G)$. If $t$ is the maximum length of a rainbow path in $G$, then $t \geqslant\left\lceil\frac{3 k}{5}\right\rceil$.

Proof. If possible suppose $t<\left\lceil\frac{3 k}{5}\right\rceil$. By Lemma 2.2, $G$ contains a rainbow cycle of length $t+1$. Let $C L$ be this cycle. Note that, $C L$ contains $(t+1)$ vertices and $(t+1)$ edges. Now, $t+1 \leqslant\left\lceil\frac{3 k}{5}\right\rceil$. Let $C L=\left\{u_{0}, u_{1}, \ldots, u_{t}, u_{0}\right\}$ and $V\left(C L^{c}\right)=V(G) \backslash V(C L)$. Let $U=\{\operatorname{color}(e): e \in E(C L)\}$ and $U^{c}=C \backslash U$, where $C$ is the set of colors used to color the edges of $G$. Let $F_{i}=\left\{z \in V\left(C L^{c}\right):\left(u_{i}, z\right) \in E(G)\right\}$.

Claim 1. $\left|F_{i}\right| \geqslant\left\lfloor\frac{2 k}{5}\right\rfloor$. Moreover, for $z \in F_{i}, \operatorname{color}\left(u_{i}, z\right) \in U$.

First part follows from the fact that the color degree of $u_{i}$ is at least $k$ as $d^{c}\left(u_{i}\right) \geqslant k$ and there are at most $\left\lceil\frac{3 k}{5}\right\rceil$ vertices in $C L$. If possible suppose $\operatorname{color}\left(u_{i}, z\right) \in U^{c}$. Now consider the path $P^{\prime}=\left\{z, u_{i}, u_{i+1}, u_{i+2}, \ldots, u_{t}, u_{0}, \ldots, u_{i-1}\right\}$. Clearly, $P^{\prime}$ is a rainbow path as color $\left(u_{i}, z\right) \in U^{c}$ and $\left\{u_{i}, u_{i+1}, u_{i+2}, \ldots, u_{t}, u_{0}, \ldots, u_{i-1}\right\}$ is already a rainbow path being a part of the rainbow cycle $C L$. Note that, the length of $P^{\prime}$ is $t+1$. This is a contradiction to the assumption that $t$ is the maximum length rainbow path in $G$. Hence Claim 1 is true.

Let $G^{\prime}=\left(V^{\prime}, E^{\prime}\right)$, where $V^{\prime}\left(G^{\prime}\right)=V(G)$ and $E^{\prime}\left(G^{\prime}\right)=E(G) \backslash\{e \in E(G)$ : color $(e) \in$ $U$ \}. Clearly, in $G^{\prime}$ there is no edge between $V(C L)$ to $V\left(C L^{c}\right)$, since by Claim 1, every such edge is colored by a color in $U$. Consider the induced subgraph on $V\left(C L^{c}\right)$ in $G^{\prime}$. Let $G^{\prime \prime}=G^{\prime}\left[V\left(C L^{c}\right)\right]$. Let $d^{c}(v) \geqslant k^{\prime}$ for every $v \in V\left(G^{\prime \prime}\right)$.

Observation 1. $k^{\prime} \geqslant\left\lfloor\frac{2 k}{5}\right\rfloor$.

Proof: Clearly $k^{\prime} \geqslant k-|U|=k-(t+1) \geqslant k-\left\lceil\frac{3 k}{5}\right\rceil \geqslant\left\lfloor\frac{2 k}{5}\right\rfloor$.

Consider the following subset $U_{0}$ of $U$, defined by $U_{0}=U_{1} \cup U_{2}$, where

$$
\begin{aligned}
& U_{1}=\left\{\operatorname{color}\left(u_{i}, u_{i+1}\right): 0 \leqslant i \leqslant\left\lceil\frac{k}{5}\right\rceil\right\}, \\
& U_{2}=\left\{\operatorname{color}\left(u_{i}, u_{i+1}\right):(t+1)-\left\lceil\frac{k}{5}\right\rceil \leqslant i \leqslant t-1\right\} \cup\left\{\operatorname{color}\left(u_{t}, u_{0}\right)\right\} .
\end{aligned}
$$


Claim 2. $\left\{\operatorname{color}\left(u_{0}, z\right): z \in F_{0}\right\} \cap U_{0}=\emptyset$.

Suppose not. Let $z \in F_{0}$ be such that $\operatorname{color}\left(u_{0}, z\right) \in U_{0}$. Without loss of generality assume that color $\left(u_{0}, z\right) \in U_{1}$. Then consider the path $P^{*}=\left(u_{\left\lfloor\frac{k}{5}\right\rfloor}, \ldots, u_{t}, u_{0}, z\right)$, which is clearly a rainbow path, since the edge of $C L$ with its color equal to $\operatorname{color}\left(u_{0}, z\right)$ is not there in this path. Also the length of $P^{*}$ is $t+1-\left\lfloor\frac{k}{5}\right\rfloor$. By Observation 1, $G^{\prime \prime}$ has minimum color degree at least $\left\lfloor\frac{2 k}{5}\right\rfloor$, and therefore by Lemma 2.1, $G^{\prime \prime}$ has a rainbow path of length at least $\left\lceil\frac{k}{5}\right\rceil$ starting from the vertex $z$, let us call this path $P^{\prime \prime}$. Clearly concatenating the path $P^{\prime \prime}$ with $P^{*}$ we get a rainbow path since colors used in $P^{*}$ belong to $U$ whereas the colors used in $P^{\prime \prime}$ belong to $U^{c}$. Moreover, the length of this rainbow path is at least $t+1$, a contradiction, to the assumption that $t$ is the length of the maximum rainbow path in $G$.

Now we complete the proof as follows: In view of Claim 2, and Claim 1, we know that $\left|F_{0}\right| \leqslant\left|U-U_{0}\right|$. But $\left|U-U_{0}\right| \leqslant\left\lceil\frac{3 k}{5}\right\rceil-\left(\left\lceil\frac{2 k}{5}\right\rceil+1\right) \leqslant\left\lceil\frac{k}{5}\right\rceil<\left\lceil\frac{2 k}{5}\right\rceil$ (since we can assume

$k \geqslant 5$ : for smaller values of $k$, the Theorem is trivially true). This is a contradiction to the first part of Claim 1.

\section{References}

[1] S. Akbari, O. Etesami, H. Mahini, M. Mahmoody. On rainbow cycles in edge colored complete graphs. Australasian Journal of Combinatorics 37, 33-42, 2007.

[2] H. Chen and X. Li. Long heterochromatic paths in edge-colored graphs. The Electronic Journal of Combinatorics. 12 (2005), \#R33.

[3] H. Gebauer, F. Mousset. On rainbow cycles and paths. (2012). arXiv:1207.0840

[4] A. Gyárás, M. Mhalla. Rainbow and orthogonal paths in factorizations of $K_{n}$. Journal of Cominatorial Designs, 18(3):167-176, 2010.

[5] M. Kano, X. Li. Monochromatic and heterochromatic subgraphs in edge-colored graphs - a survey. Graphs Combin. 24 (2008), 237 - 263. 